\title{
Debris of Asteroid Disruptions Close to the Sun
}

\author{
Ye, Quanzhi
}

2019-03-10

Ye , Q \& Granvik, M 2019 , ' Debris of Asteroid Disruptions Close to the Sun ' , Astrophysical Journal , vol. 873 , no. 2 , 104 . https://doi.org/10.3847/1538-4357/ab05ba

http://hdl.handle.net/10138/303513

https://doi.org/10.3847/1538-4357/ab05ba

unspecified

publishedVersion

Downloaded from Helda, University of Helsinki institutional repository.

This is an electronic reprint of the original article.

This reprint may differ from the original in pagination and typographic detail.

Please cite the original version. 


\title{
Debris of Asteroid Disruptions Close to the Sun*
}

\author{
Quanzhi Ye (叶泉志) $)^{1,2,3}$ (1) and Mikael Granvik ${ }^{4,5}$ (1) \\ ${ }^{1}$ Division of Physics, Mathematics and Astronomy, California Institute of Technology, Pasadena, CA 91125, USA; qye@ caltech.edu \\ ${ }^{2}$ Infrared Processing and Analysis Center, California Institute of Technology, Pasadena, CA 91125, USA \\ ${ }^{3}$ Department of Physics and Astronomy, The University of Western Ontario, London, Ontario N6A 3K7, Canada \\ ${ }^{4}$ Division of Space Technology, Luleå University of Technology, Box 848, SE-98128 Kiruna, Sweden \\ ${ }^{5}$ Department of Physics, P.O. Box 64, FI-00014 University of Helsinki, Finland \\ Received 2018 December 26; revised 2019 January 24; accepted 2019 February 4; published 2019 March 11
}

\begin{abstract}
The under-abundance of asteroids on orbits with small perihelion distances suggests that thermally driven disruption may be an important process in the removal of rocky bodies in the solar system. Here we report our study of how the debris streams arise from possible thermally driven disruptions in the near-Sun region. We calculate that a small body with a diameter $\gtrsim 0.5 \mathrm{~km}$ can produce a sufficient amount of material to allow the detection of the debris at the Earth as meteor showers, and that bodies at such sizes thermally disrupt every $\sim 2 \mathrm{kyr}$. We also find that objects from the inner parts of the asteroid belt are more likely to become Sun-approachers than those from the outer parts. We simulate the formation and evolution of the debris streams produced from a set of synthetic disrupting asteroids drawn from Granvik et al.'s near-Earth object population model, and find that they evolve 10-70 times faster than streams produced at ordinary solar distances. We compare the simulation results to a catalog of known meteor showers on Sun-approaching orbits. We show that there is a clear overabundance of Sun-approaching meteor showers, which is best explained by a combining effect of comet contamination and an extended disintegration phase that lasts up to a few thousand years. We suggest that a few asteroid-like Sun-approaching objects that brighten significantly at their perihelion passages could, in fact, be disrupting asteroids. An extended period of thermal disruption may also explain the widespread detection of transiting debris in exoplanetary systems.
\end{abstract}

Key words: meteorites, meteors, meteoroids - minor planets, asteroids: general - protoplanetary disks

Supporting material: animation

\section{Introduction}

Near-Earth object (NEO) population models have predicted the existence of numerous Sun-approaching asteroids (e.g., Bottke et al. 2002; Greenstreet et al. 2012), but after a few decades of rigorous NEO search, few have been found. It has been proposed that thermally driven "super-catastrophic" disruption is responsible for an efficient removal of asteroids that reach a few tenths of an astronomical unit (Granvik et al. 2016), but such a disruption has not been directly observed.

Rather than catching an asteroid disruption in action, it may be easier to detect the end product of a disruption. A cloud of dust debris resulting from a complete disintegration continues to orbit the Sun on the orbit of the disintegrated asteroid. A range of different effects such as ejection velocity, planetary perturbations, and radiation pressure gradually disperse the dust cloud, most noticeably along the orbit, forming a dust (meteoroid) "stream" (see Olsson-Steel 1987; Brown \& Jones 1998; Williams et al. 2004). The stream continues to disperse over time, eventually blending into the interplanetary meteoroid background (e.g., Cremonese et al. 1997). Until its dispersal, the meteoroid stream can be detected as a meteor shower on the Earth, if it is on an Earth-crossing orbit and dense enough to stand out from the interplanetary meteoroid background.

Optical and radio meteor surveillance systems that have been in operation over the past few decades have detected a handful of meteoroid streams on Sun-approaching orbits (e.g., Brown et al. 2008; Jenniskens et al. 2016b). A few prominent streams,

\footnotetext{
* Data and codes that generate the figures and main results of this work are publicly available on Zenodo (doi:10.5281/zenodo.2547298) and GitHub (https://github.com/Yeqzids/near-sun-disruptions).
}

such as the well-known Geminid meteoroid stream, are easily detectable and have been studied for decades (e.g., Denning 1893; Plavec 1950; Whipple 1983; Jones et al. 2016; Hui \& Li 2017; Ryabova \& Rendtel 2018, and many others). However, most of the Sun-approaching streams are weakly active and have not received a lot of attention (Ye 2018). Many of these streams do not have identifiable parents, raising questions about their formation mechanism.

Here, we present an investigation of the population of Sunapproaching meteoroid streams, with the goal being to critically examine the hypothesis that some (or most) of these streams were produced by thermally driven disruptions of asteroids with small perihelion distances $(q)$. The investigation is divided into two complementary parts: on one hand, we will predict the number of small- $q$ meteoroid streams formed by thermally driven disruptions by utilizing an NEO population model (Section 2); on the other hand, we will examine the small- $q$ streams that are actually observed (Section 3). Results from these two parts will be compared to each other and discussed in Section 4.

\section{Predicted Characteristics of Thermally Driven Streams}

\subsection{Size of the Parent and the Detectability of the Resulting Meteoroid Streams ${ }^{6}$}

Meteor showers are essentially local overdensities of meteor radiants; therefore, meteoroid streams that are detectable need to stand out against the interplanetary meteoroid background. Since the flux intensity of a stream is directly related to the

\footnotetext{
6 The Jupyter notebook that shows the calculation of the numbers mentioned in this section can be found at https://github.com/Yeqzids/near-sundisruptions/blob/master/nb/stream_mass.ipynb.
} 
production rate of the parent, a disrupting parent needs to be massive enough to produce enough dust to supply a detectable stream. Therefore, the first question is the critical parent size needed to produce a detectable stream.

Radar and optical techniques are the most widely used methods to detect meteors (Jenniskens 2017), therefore, in this work, we focus on the regimes explored by these two techniques. The detection limit of typical meteor radars are in the range of $\sim 10^{-3}-10^{-2} \mathrm{~km}^{-2} \mathrm{hr}^{-1}$, appropriate to meteoroid sizes down to $1 \mathrm{~mm}$ (Ye et al. 2016a, 2016b), while for typical video systems, $10^{-5}-10^{-4} \mathrm{~km}^{-2} \mathrm{hr}^{-1}$, appropriate to meteoroid sizes down to $1 \mathrm{~cm}$ (Jenniskens et al. 2016a, 2016b). These two numbers translate to a Zenith Hourly Rate of $\lesssim 1$ using the relation derived by Koschack \& Rendtel (1990, Section 10), assuming a typical duration of stream activity of a couple days, a collection area (i.e., the area of atmosphere that one system monitors) of $10^{4} \mathrm{~km}^{2}$, a typical meteoroid mass index of 1.6 (Blaauw et al. 2011), and a system uptime of $6 \mathrm{hr}$ each day, which is in the same ballpark as the typical background flux observed by visual observers.

The mass of a meteoroid stream can be calculated following the derivation of Hughes \& McBride (1989):

$$
M=\frac{f \pi t^{2} V_{\mathrm{E}}^{2} I \sin ^{2} \epsilon V_{\mathrm{H}} P}{4 V_{\mathrm{G}}},
$$

where $f \sim 10$ is a dimensionless factor that accounts for the shape of the stream (McIntosh \& Jones 1988), $t$ is the duration of the meteor shower at the Earth in seconds, $V_{\mathrm{E}}=29,700 \mathrm{~m} \mathrm{~s}^{-1}$ is the orbital speed of the Earth, $I$ is the mass influx at the Earth in $\mathrm{kg} \mathrm{m}^{-2} \mathrm{~s}^{-1}, \epsilon$ is the angle between the Earth's path and the orbit of the stream, $V_{\mathrm{H}}, V_{\mathrm{G}}$ is the heliocentric and geocentric speed of the meteoroids, respectively, and $P$ is the orbital period of the meteoroids in seconds. Recognizing that such a calculation is only to be taken at an order of magnitude level, we take $\sin \epsilon \sim 1$, $V_{\mathrm{H}} / V_{\mathrm{G}} \sim 1$, average meteoroid density of $2000 \mathrm{~kg} \mathrm{~m}^{-3}$ (Rotundi et al. 2015), and the flux and meteoroid sizes discussed above, we derive $M \sim 10^{11} \mathrm{~kg}$ as the minimum mass of the progenitor that is needed for the detection of the resulting meteoroid stream by contemporary meteor-detection networks. This number corresponds to a progenitor diameter of $\sim 0.5 \mathrm{~km}$ (assuming a density of $2000 \mathrm{~kg} \mathrm{~m}^{-3}$, see Carry 2012). The stream mass derived from different observations can differ by a factor of 10 from the mean (Ryabova 2017), which translates to a factor of $\sim 2$ in the uncertainty of the progenitor size.

\subsection{Rate of Thermally Driven Disruptions}

How often does thermally driven disruption occur? With some necessary simplification of the problem, this can be estimated using an NEO population model. Here we use the Granvik et al. (2018) model, which considered the effect of thermally driven disruption of NEOs. We start from the predicted flux of NEOs from known sources of NEOs in the main asteroid belt, and then multiply these fluxes with the predicted likelihood that an asteroid from a specific region of the main asteroid belt would reach the critical heliocentric distance from the Sun $(0.058$ au for kilometer-sized asteroids, as derived by Granvik et al. 2016). Finally, we sum up the products to arrive at a total rate of thermal disruptions of $550 \pm 30 \mathrm{Myr}^{-1}$, where the error accounts for uncertainties in the flux of NEOs from different parts of the asteroid belt.
Table 1

Probabilities That Asteroids from Different Escape Regions in the Asteroid Belt End Up on Sun-approaching Orbits $\left(P_{\text {sungrazer }}\right)$ As Well As the Timescale That They Have $q<0.14$ au Prior to Disruption at $q=0.058$ au $\left(t_{\text {sungrazer }}\right)$

\begin{tabular}{lcc}
\hline \hline Escape Region & $P_{\text {sungrazer }}$ & $t_{\text {sungrazer }}$ \\
\hline Hungaria & $78 \%$ & $13,000 \mathrm{yr}$ \\
Phocaea & $89 \%$ & $1400 \mathrm{yr}$ \\
$\nu_{6}$ complex & $81 \%$ & $13,000 \mathrm{yr}$ \\
3:1J complex & $74 \%$ & $2800 \mathrm{yr}$ \\
5:2J complex & $22 \%$ & $1500 \mathrm{yr}$ \\
2:1J complex & $22 \%$ & $700 \mathrm{yr}$ \\
\hline
\end{tabular}

Note. Escape regions with the format of $X: Y \mathrm{~J}$ stand for $X: Y$ mean-motion resonance with Jupiter.

The result of our calculation is summarized in Table 1. We observe two things: (1) asteroids from the inner main-belt region (Hungarias, Phocaeas, low-inclination $\nu_{6}$ objects) are more likely to become Sun-approachers; and (2) asteroids from the inner mainbelt region also tend to spend a longer time as Sun-approachers compared to outer main-belt asteroids. A direct implication of these two features is that meteoroid streams from the inner belt should dominate the thermally driven streams, while streams with semimajor axes $a$ compatible with an origin in the outer belt are statistically unlikely to be thermally driven disrupted asteroids.

\subsection{Distribution and Behavior of Thermally Produced Streams}

\subsubsection{Simulation Setup}

We randomly select $1 \%$ (608 out of 60,727) of the test asteroids in the orbital integrations by Granvik et al. (2016) that have perihelion distances within the critical disruption distance of $q_{*}=0.058 \mathrm{au}$, representing the population of thermally disrupting asteroids (Figure 1). We note that the $(a, \sin i)$ distribution covers the essential parts of the phase space whereas the $(a, e)$ distribution is limited to orbits with small perihelion distances, as it should be. We then generate a total of 500 particles for each test asteroid at their respective perihelion point, mimicking the thermally driven disintegration of asteroids. The size distribution of these particles follows a size range of $a \subseteq\left[5 \times 10^{-4}, 5 \times 10^{-2}\right] \mathrm{m}$, a meteoroid bulk density of $2000 \mathrm{~km} \mathrm{~m}^{-3}$, and a continuous size distribution of $d N / d a \propto a^{-q}$, where $q=3.6$, as suggested by telescopic observations (e.g., Fulle 2004; Ye et al. 2016c).

We integrate these subparticles for $10 \mathrm{kyr}$ using the BulirschStoer integrator (Stoer \& Bulirsch 2013) embedded in a tailored MERCURY6 N-body simulator (Chambers 1999; Ye et al. 2016a). The code accounts for gravitational perturbations by major planets (Mercury through Neptune with the Earth-Moon system represented by a single point of mass), radiation pressure, Poynting-Robertson drag, as well as perihelion shift due to general relativity. The orbital elements of all test particles are recorded with a cadence of $1 \mathrm{yr}$. The animation in Figure 2 depicts the formation, evolution, and final dispersion of a meteoroid stream.

The simulation is run two times with different ejection models: the first one with Crifo \& Rodionov's (1997) cometary ejection model $;^{7}$ and the second one with an ejection model

\footnotetext{
Readers may wonder about choosing Crifo \& Rodionov's (1997) model over the Whipple (1951) model. These two models were derived from different approaches but produced similar end results. As we will show in the following section, the choice of ejection model does not have significant impact on our conclusion, and is hence unimportant.
} 

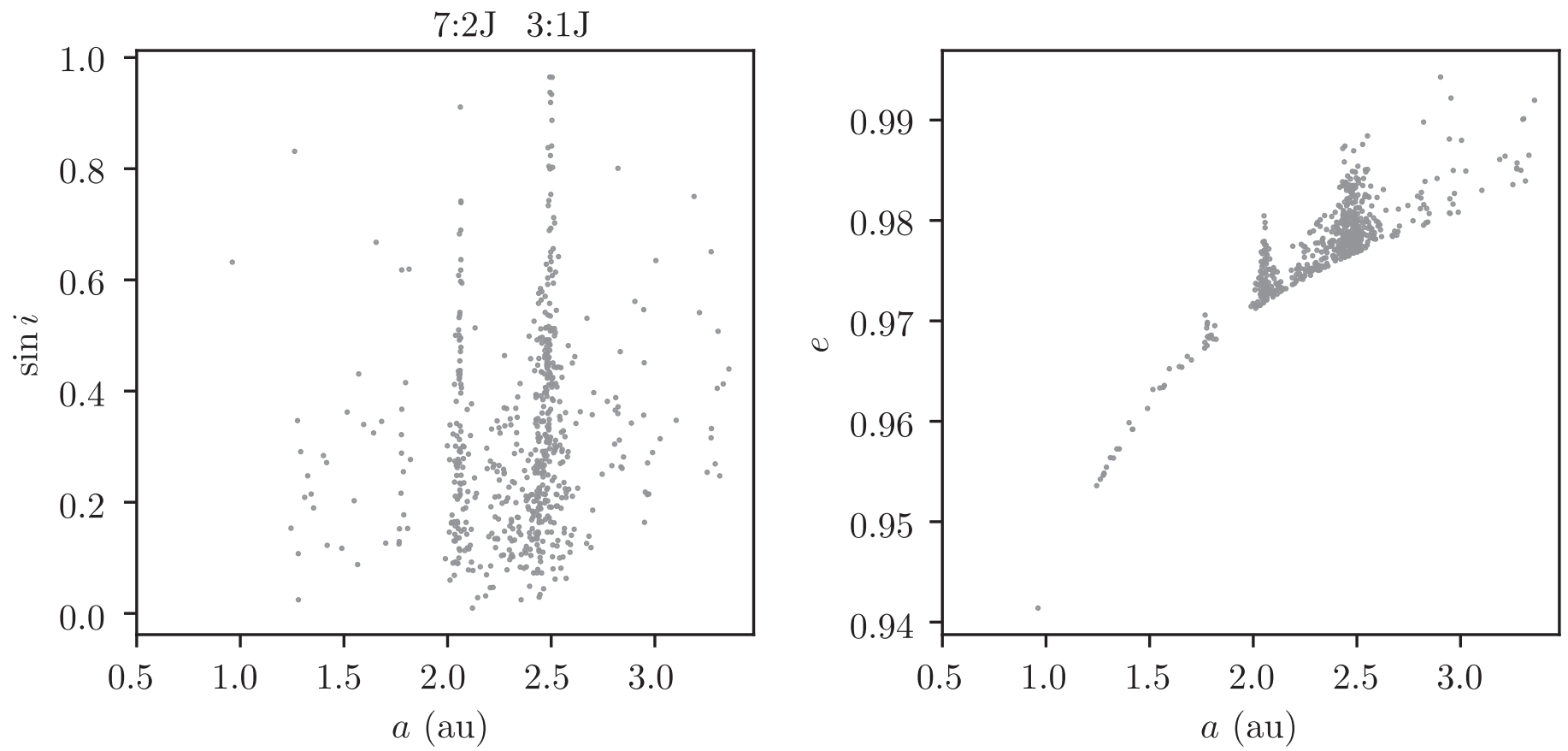

Figure 1. $a, e$, and $\sin i$ of the synthetic asteroids drawn from Granvik et al.'s (2016) NEO population model. The overdensities produced by 7:2J (which coincides with Hungaria, Phocaea, and $\nu_{6}$ at low inclinations) and 3:1J resonance are marked. The Jupyter notebook for this figure is available at https://github.com/Yeqzids/ near-sun-disruptions/blob/master/nb/test_asteroids.ipynb.

that assumes gravitational escape ejection (i.e., eject at escape velocity). This is due to the poorly understood ejection scheme of a thermally driven disruption, but it is reasonable to expect that the ejection speed should be somewhere between the one set by the cometary model and the one set by the gravitational escape model. Here we note that C/2015 D1 (Solar and Heliospheric Observatory $(\mathrm{SOHO})$ ), a Sun-approaching comet that disintegrated during its perihelion passage in early 2015 with excess thermal stress as the likely cause, showed morphology consistent with comet-like ejection (Hui et al. 2015); however, C/2015 D1 is of cometary origin and the lesson it provided may not be applicable to asteroids.

We also acknowledge that the information provided by 500 particles per test asteroid is somewhat limited, considering that many meteoroid stream simulations account for thousands of particles. We are mainly limited by the high computational cost of the simulation: the full simulation of 608 meteoroid streams over $10 \mathrm{kyr}$ with two sets of input parameters takes about 50 CPU years on a single $2.2 \mathrm{GHz}$ AMD Opteron CPU, though with distributed computing we are able to complete our simulation within a few months. The exact numbers derived for each stream become less important as we collectively examine a large set of streams, which we believe is sufficient for deriving a broad, global picture of asteroid disruptions close to the Sun.

\subsubsection{Calculation of Stream Formation, Dispersion, and Visible Timescale}

Following the definition in our earlier work (Ye et al. 2016a), we define the stream formation time as the time taken to the point that the standard deviation of the mean anomalies of the test particles reach $60^{\circ}$ (i.e., the $3 \sigma$ limits cover the entire orbit if the distribution of mean anomalies is Gaussian), while the stream-dispersal timescale is defined as the time it takes for $50 \%$ of the test particles to lose stream coherency. The stream coherency is defined using the Southworth \& Hawkins (1963) decoherence parameter $(D)$, which is defined as:

$$
\begin{aligned}
D_{A, B}^{2}= & \left(q_{B}-q_{A}\right)^{2}+\left(e_{B}-e_{A}\right)^{2} \\
& +\left(2 \sin \frac{I}{2}\right)^{2}+\left[\left(e_{A}+e_{B}\right) \sin \frac{\Pi}{2}\right]^{2},
\end{aligned}
$$

where

$$
\begin{aligned}
I= & \arccos \left[\cos i_{A} \cos i_{B}\right. \\
& \left.+\sin i_{A} \sin i_{B} \cos \left(\Omega_{A}-\Omega_{B}\right)\right]
\end{aligned}
$$

and

$$
\begin{aligned}
\Pi= & \omega_{A}-\omega_{B}+2 \arcsin \\
& \times\left(\cos \frac{i_{A}+i_{B}}{2} \sin \frac{\Omega_{A}-\Omega_{B}}{2} \sec \frac{I}{2}\right)
\end{aligned}
$$

and the subscripts $A$ and $B$ refer to the two test particles being compared, $q$ is the perihelion distance in astronomical units, $e$ is the eccentricity, $i$ is the inclination, $\Omega$ is the longitude of ascending node, and $\omega$ is the argument of perihelion. The sign of the arcsin term in the equation for $\Pi$ switches over if $\left|\Omega_{A}-\Omega_{B}\right|>180^{\circ}$. We adopt an empirical cutoff of $D=0.1$ used by many of the past works (e.g., Drummond 1981; Jopek 1993), noting that cutoffs found by more rigorous tests (e.g., Fu et al. 2005; Moorhead 2016) are not substantially different from $D=0.1$.

Streams cannot be detected unless they intercept the Earth's orbit; therefore, we also need to calculate their "visible time" at Earth. A stream is considered to be "visible" if the spread of either or both of the heliocentric distances of their ascending/descending 

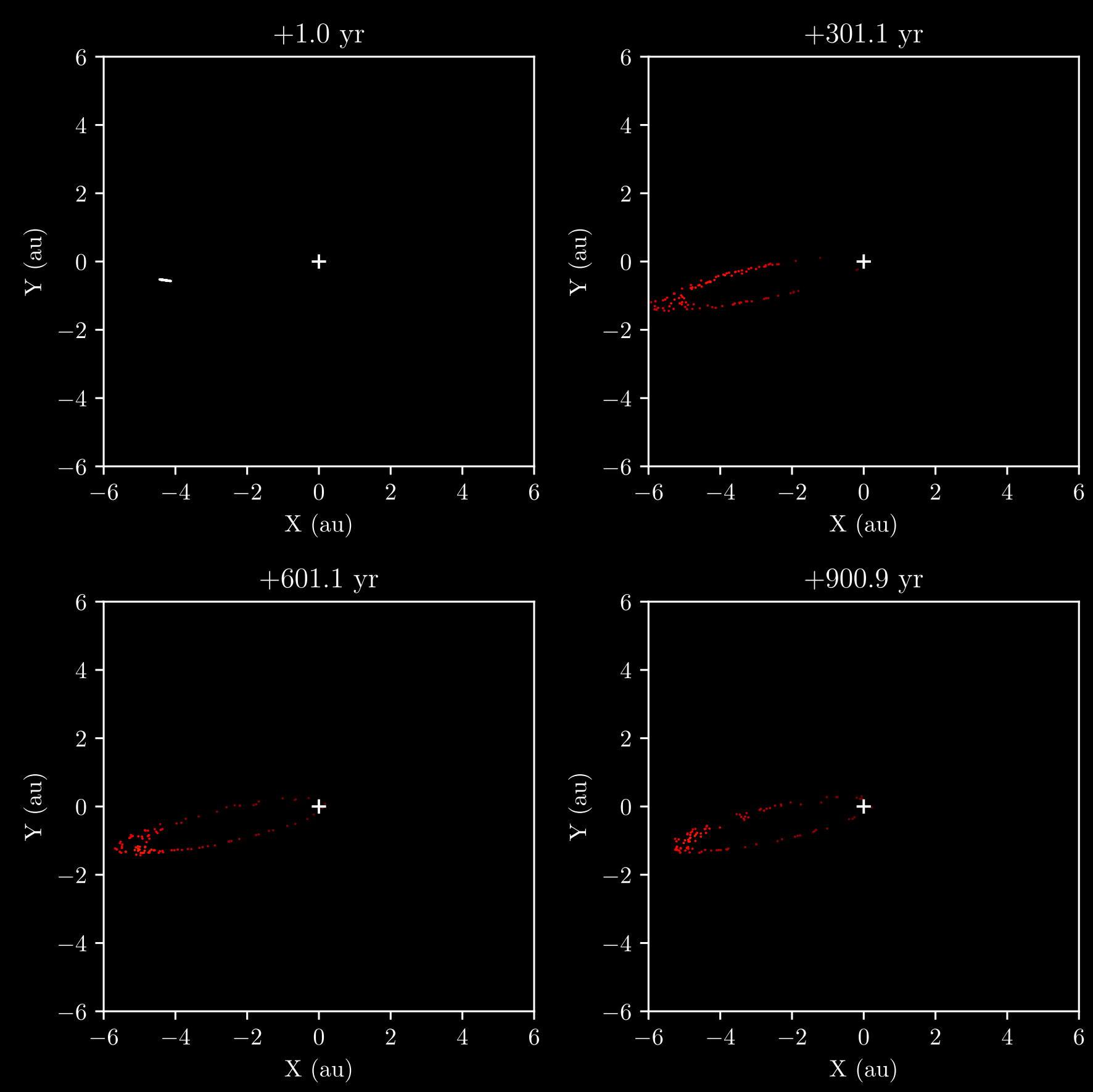

Figure 2. Disruption of four randomly selected synthetic asteroids, assuming dust ejection at gravitational escape speed of a kilometer-sized body. The Sun is marked by a white cross. An animation of the full integration is available in the online journal. The Jupyter notebook for this figure/animation is available at https://github. com/Yeqzids/near-sun-disruptions/blob/master/nb/test_asteroids.ipynb.

(An animation of this figure is available.)

nodes encompass 1 au (Earth's average distance to the Sun) before it disperses. We then sum up the time that stream is visible to derive the "visible timescale" at the Earth.

\subsubsection{Results}

We find that results from different ejection models are not dramatically different: the "cometary" model predicts a median formation and dispersion timescale of $6 \pm 1 \mathrm{yr}$ and $170_{-130}^{+1500} \mathrm{yr}$, while the gravitational escape model predicts $8 \pm 1 \mathrm{yr}$ and $280_{-240}^{+2000} \mathrm{yr}$; both ejection models predict that $\sim 75 \%$ of the simulated streams will be visible at some point during their lifetime, for a median total time of $40_{-25}^{+140} \mathrm{yr}^{8}$ (Error bars indicate $1 \sigma$ probability interval.) The formation and dispersion

\footnotetext{
The Jupyter notebook for this calculation is available at https://github.com/ Yeqzids/near-sun-disruptions/blob/master/nb/formation_and_dispersion_ timescales.ipynb.
} 
timescales are significantly shorter than for typical (nonSun-approaching) meteoroid streams, which take $400 \pm 80 \mathrm{yr}$ to form and $3000 \pm 300 \mathrm{yr}$ to disperse (Ye et al. 2016a).

Figure 3 shows the dependence of various timescales to orbital elements $a$ and $T_{\mathrm{J}}$. No clear dependence is seen between the formation timescale and dynamical properties of the parent. The dispersion and visible timescales, on the other hand, show a clear dependence on whether the stream is on a Jupiterapproaching orbit (which can be measured by $T_{\mathrm{J}}$ ) or close to resonances. Streams that are dynamically decoupled from Jupiter (i.e., $T_{\mathrm{J}}>3$ ) are longer-lived than those that are not, except for the streams that originate from parents close to resonances (Hungaria, Phocaea, 7:2J, or 3:1J complex).

One key takeaway from Figure 3 is that thermally produced meteoroid streams have very short visibility at the Earth, due to the fact that they are mostly shortly lived and are dynamically quickly evolving. Given a typical visible timescale of $40 \mathrm{yr}$, if we consider the disruption rate of kilometer-sized NEOs that we derived in Section 2.2, which is $\sim 0.6 \mathrm{kyr}^{-1}$, we can immediately see that the chance of seeing a thermally produced meteoroid stream is close to zero.

\section{Known Sun-approaching Meteoroid Streams}

The official catalog of meteor showers is managed by the International Astronomical Union's Meteor Data Center (IAUMDC; Jopek \& Jenniskens 2011; Jopek \& Kaňuchová 2014, 2017). Under the current rule of meteor shower nomenclature, a previously unreported stream will be added to the Working List of Meteor Showers, but will only be considered for "established" status when it is independently redetected by other observers (Jopek \& Kaňuchová 2014; Janches et al. 2018). Promotion of status and assignment of official names is overseen by the IAU F1 Commission during the triennial General Assemblies (GA) meeting. Currently, the most recent version of the IAUMDC catalog was reviewed at the GA meeting in 2018 August and dated 2018 November 9. This version has a total of 932 meteor showers with 112 of them considered to be established and will be used in the analysis in this work. Since showers on the Working List are to be considered provisional detections and thus subjected to removal if they cannot be confirmed, we will focus on the established showers for now.

Table 2 lists all 22 established Sun-approaching meteoroid streams in the IAUMDC catalog that have perihelion distance $q<0.15 \mathrm{au}$. A loose cutoff at $0.15 \mathrm{au}$ is chosen because the Geminids $/ \mathrm{GEM}^{9}$ stream, the prominent candidate for thermally produced streams, has $q=0.14$ au. The 22 streams have the following characteristics:

1. Eight have $T_{\mathrm{J}}<2$ (i.e., compatible with orbits of Halleytype and long-period comets). Even though only one of them has a proposed parent body that is a comet (KLE and C/1917 F1, see Vereš et al. 2011; Neslušan \& Hajduková 2014), they are most likely to be produced by comets based on their dynamical properties.

2. Four have $2<T_{\mathrm{J}}<3$. This type of orbit is compatible with Jupiter-family comets and asteroidal interlopers (Levison \& Duncan 1994; Jewitt et al. 2015; Hsieh \& Haghighipour 2016). Among these showers, SDA has a proposed parent (the $96 \mathrm{P} / \mathrm{Machholz}$ complex) that is

\footnotetext{
9 For readability, from now on we will use the IAUMDC three-letter code to refer to a shower. The full name of the shower is listed in Table 2.
}

considered to be rather definitive (e.g., Abedin et al. 2017, 2018).

3. Ten have $T_{\mathrm{J}}>3$ that are dynamically in the asteroid regime. The $96 \mathrm{P} / \mathrm{Machholz}$ Complex is thought to be responsible for ARI (Bruzzone et al. 2014; Abedin et al. 2017, 2018), a stream that is near the borderline of $T_{\mathrm{J}}=3$, while (3200) Phaethon and (155140) 2005 UD are widely believed to be the parents of the Geminids and Daytime Sextantids. IAUMDC lists three comets found by the $\mathrm{SOHO}$ probe as likely parents for NOC, NZC, and OCE, but no reference is provided and we cannot find any published papers that discussed these linkages; additionally, the orbits of these $\mathrm{SOHO}$ comets are poorly known due to the extremely short arc (less than a day for two of the comets, a week for the third); therefore, the linkages are doubtful. In either case, there are four to seven dynamically asteroidal streams that have no known parent bodies associated with them.

\subsection{Ages of Meteoroid Streams}

\subsubsection{Age-Width-T Map}

Aside from the orbit, which gives us an idea of the dynamical properties of a stream, another piece of useful information that we can get from meteor observations is the age of the stream. As noted in Section 1, the stream age correlates to the dispersion of the stream, which can be measured through the width of the stream as the Earth travels through the orbital intersection, i.e., the duration of the shower activity. Although an accurate determination of the age requires knowledge of a number of poorly constrained parameters, such as how meteoroids are ejected as well as their physical and mechanical properties, and is therefore difficult if not impossible to carry out, a crude comparison between the observed shower width and dynamical models is usually sufficient to broadly constrain the age of a stream. This has been done for many meteoroid streams including Quadrantids, Perseids, Geminids, and others (e.g., Williams \& Wu 1993; Brown \& Jones 1998; Ryabova 1999; Abedin et al. 2018).

However, this method requires a priori knowledge of the orbit of the parent, because the dynamics of the stream and the parent evolve over time and are not fully correlated with each other. Most of the streams in Table 2 do not have known parents and, as we have shown earlier, Sun-approaching streams evolve faster than typical meteoroid streams. Therefore, we must seek a different path to achieve our goal.

Our solution is to reuse the $N$-body simulations completed in Section 2 to map the dependence between age, stream width, and $T_{\mathrm{J}} . T_{\mathrm{J}}$ is one of the variables here because the perturbation from Jupiter is a dominant factor of the dynamical evolution of meteoroid streams. The major benefit of this method is that it bypasses the need to know the parent's orbit, because the set of possible orbits of the parent is already captured by the NEO population model.

We focus on the $14 T_{\mathrm{J}}>2$ streams in Table 2, as we are most interested in the thermally driven disruptions of asteroids and the NEO population model is not applicable to $T_{\mathrm{J}}<2$ objects. To generate the age-width- $T_{\mathrm{J}}$ map, we first determine the width of the stream and the mean $T_{\mathrm{J}}$ for each simulated stream at each time step. The stream width is defined as the $90 \%$ width of the longitude of the ascending node $(\Omega)$, the point where the particle passes the ecliptic plane 

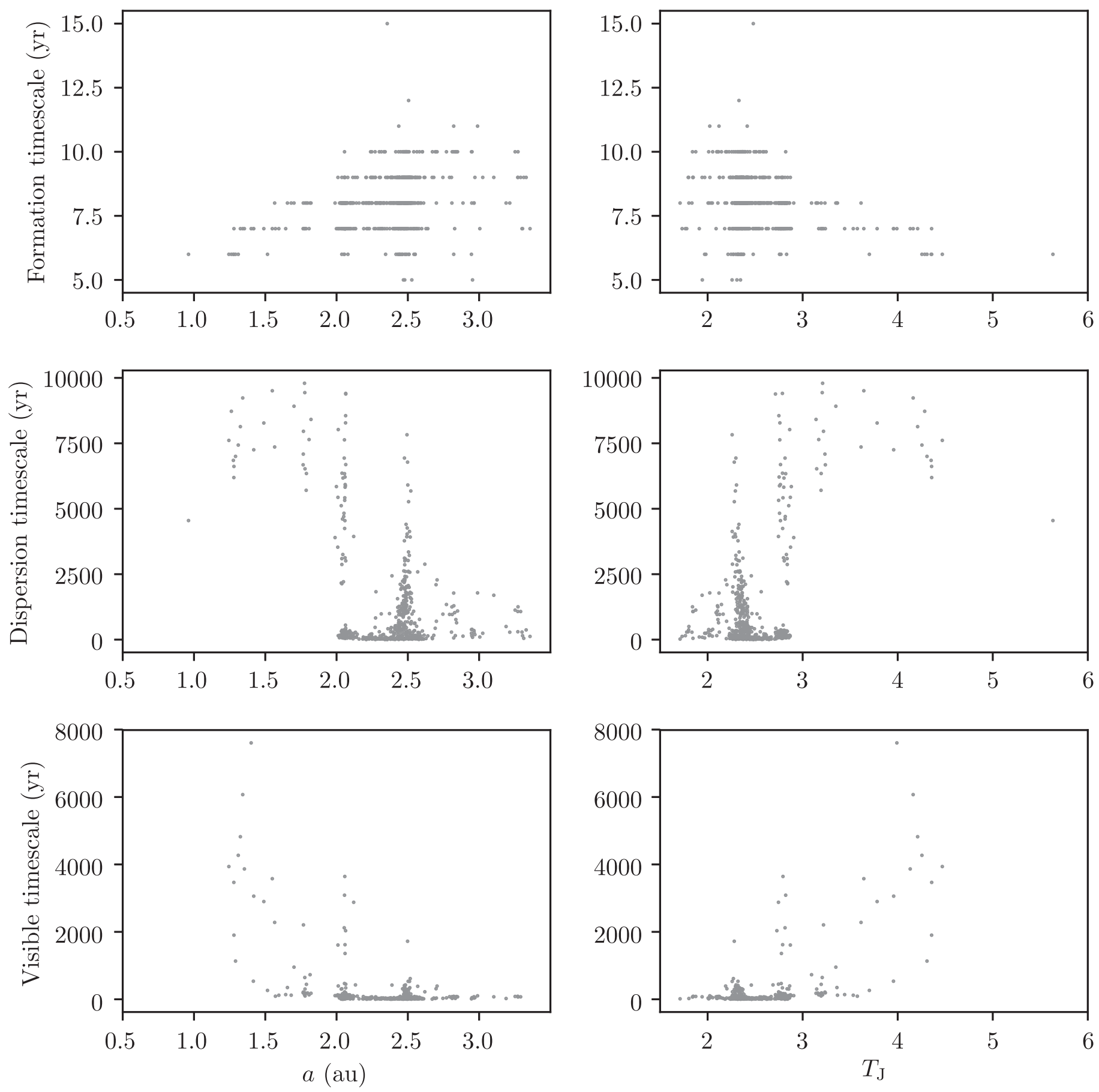

Figure 3. Different timescales of the simulated meteoroid streams as well as their dependence on $a$ and $T_{\mathrm{J}}$ of the streams. Distributions are drawn from the simulations that assume ejection at gravitational escape speed. The formation timescales (the two upper panels) look discrete as they are comparable to the output interval of the integration (1 yr). The Jupyter notebook for this figure is available at https://github.com/Yeqzids/near-sun-disruptions/blob/master/nb/timescales_a_tj.ipynb.

(i.e., the plane of Earth's orbit). The 90th percentile is used to reject the few random particles that might have been gravitationally scattered during the integration. Mean $T_{\mathrm{J}}$ is defined as the mean of the $T_{\mathrm{J}}$ values of all particles. We calculate the mean stream width and $T_{\mathrm{J}}$ for every time step until the stream has lost $50 \%$ of the particles (due to solar impact or ejection from the solar system) or the end of the integration has been reached. To increase the clarity of the map, we apply a Gaussian filter with $\sigma=3.0$ (an arbitrarily chosen number) to the derived age-width- $T_{\mathrm{J}}$ map to remove sharp gradients resulting from limited statistics.
The aforementioned procedure is applied to the simulation results obtained from both the cometary ejection model and the gravitational escape ejection model. The raw input as well as the final processed age-width- $T_{\mathrm{J}}$ map are shown in Figures 4 and 5. Our primary goal here is to validate the accuracy of this map and use it to estimate the ages of the streams in Table 2, but prior to validation we do note the following general features of the map:

1. As expected, streams at higher $T_{\mathrm{J}}$ are generally longerlived than those at lower $T_{\mathrm{J}}$. 
Table 2

Established Showers in the IAUMDC Database with $q<0.15$ au

\begin{tabular}{|c|c|c|c|c|c|c|c|c|c|c|c|}
\hline \multirow{2}{*}{ IAUMDC code/name } & \multirow{2}{*}{$\lambda_{\odot}$} & \multicolumn{2}{|c|}{ Radiant } & \multirow{2}{*}{$\begin{array}{c}V_{\mathrm{G}} \\
\left(\mathrm{km} \mathrm{s}^{-1}\right)\end{array}$} & \multirow{2}{*}{$\begin{array}{c}a \\
(\mathrm{au})\end{array}$} & \multirow{2}{*}{$e$} & \multirow[t]{2}{*}{$i$} & \multirow{2}{*}{$T_{\mathrm{J}}$} & \multirow[t]{2}{*}{ Tech. } & \multirow{2}{*}{$\begin{array}{c}\text { Est. Age } \\
\text { (kyr) }\end{array}$} & \multirow{2}{*}{ Parent Body } \\
\hline & & R.A. & Decl. & & & & & & & & \\
\hline AAN $\alpha$ Antliids & 313.1 & $160^{\circ} 7$ & -11.9 & 43.9 & 2.4 & 0.94 & 62.7 & 2.5 & $\mathrm{O}+\mathrm{R}$ & 4 & \\
\hline ARI Daytime Arietids & 76.2 & 42.5 & +24.0 & 38.2 & 1.9 & 0.95 & 24.6 & 3.2 & $\mathrm{O}+\mathrm{R}$ & 5 & 96P/Machholz complex \\
\hline CTA $\chi$ Taurids & 220.5 & 63.1 & $+25^{\circ} .4$ & 41.6 & 4.9 & 0.98 & 13.7 & 1.4 & $\mathrm{O}+\mathrm{R}$ & $\ldots$ & \\
\hline DLT Daytime $\lambda$ Taurids & 85.5 & 56.7 & +11.5 & 36.4 & 1.6 & 0.93 & 23.2 & 3.7 & $\mathrm{O}+\mathrm{R}$ & 5.5 & \\
\hline DSX Daytime Sextantids & $186^{\circ} 7$ & $155^{\circ} 0$ & -1.6 & 31.8 & 1.1 & 0.86 & 22.5 & 5.1 & $\mathrm{O}+\mathrm{R}$ & $6-7$ & (155140) 2005 UD \\
\hline EPG $\epsilon$ Pegasids & 108.6 & $329^{\circ} .9$ & +14.5 & 28.6 & 0.7 & 0.78 & 49.7 & 7.3 & $\mathrm{O}+\mathrm{R}$ & $6-9$ & \\
\hline EPR $\epsilon$ Perseids & 91.1 & 55.7 & $+37^{\circ} .6$ & 44.3 & 7.3 & 0.98 & 57.1 & 1.0 & $\mathrm{O}+\mathrm{R}$ & $\ldots$ & \\
\hline GEM Geminids & 261.6 & $113 .^{\circ} 0$ & +32.3 & 34.5 & 1.4 & 0.90 & 23.5 & 4.2 & $\mathrm{O}+\mathrm{R}$ & $6.5-7$ & (3200) Phaethon \\
\hline JLE January Leonids & 282.5 & $148^{\circ} .1$ & +23.9 & 52.1 & 5.7 & 0.99 & $105^{\circ} 8$ & 0.8 & $\mathrm{O}+\mathrm{R}$ & $\ldots$ & \\
\hline KLE Daytime $\kappa$ Leonids & 182.1 & $162^{\circ} .2$ & $+15^{\circ} 3$ & 43.4 & 20.2 & 0.99 & $25^{\circ} 0$ & 0.9 & $\mathrm{R}$ & $\ldots$ & C/1917 F1 (Mellish) \\
\hline NDA Northern $\delta$ Aquariids & 140.6 & $345^{\circ} 6$ & +1.0 & 39.2 & 2.2 & 0.96 & $22 .^{\circ} 0$ & 2.8 & $\mathrm{O}+\mathrm{R}$ & $4-5$ & \\
\hline NOC Northern Daytime $\omega$ Cetids & 46.6 & 5.7 & $+17^{\circ} .6$ & 34.9 & 1.3 & 0.91 & $38^{\circ} .1$ & 4.7 & $\mathrm{O}+\mathrm{R}^{\mathrm{a}}$ & $6.5-7$ & See note ${ }^{\mathrm{b}}$ \\
\hline NOO November Orionids & $245^{\circ} 9$ & $89^{\circ} 1$ & +15.4 & 43.1 & 11.2 & 0.99 & 36.2 & 0.8 & $\mathrm{O}+\mathrm{R}$ & $\ldots$ & \\
\hline NZC Northern June Aquilids & 99.1 & 308.4 & -5.1 & 37.8 & 1.7 & 0.93 & 38.8 & 3.4 & $\mathrm{O}+\mathrm{R}$ & 6 & See note ${ }^{c}$ \\
\hline OCE Southern Daytime $\omega$ Cetids & 46.4 & 20.7 & $-5^{\circ} 6$ & 36.7 & 1.6 & 0.92 & $35^{\circ} 1$ & 3.5 & $\mathrm{R}$ & 6 & See note ${ }^{b}$ \\
\hline PAU Piscis Austrinids & 131.2 & 350.2 & -22.1 & 44.0 & 4.4 & 0.97 & 58.6 & 1.5 & $\mathrm{O}+\mathrm{R}$ & $\ldots$ & \\
\hline SDA Southern $\delta$ Aquariids & 126.8 & $336^{\circ} 4$ & $-16^{\circ} 1$ & 40.9 & 2.6 & 0.97 & 28.7 & 2.3 & $\mathrm{O}+\mathrm{R}$ & 4 & 96P/Machholz complex \\
\hline SSE $\sigma$ Serpentids & 275.5 & 243.5 & -1.7 & 43.5 & 2.7 & 0.93 & $62{ }^{\circ} 1$ & 2.4 & $\mathrm{O}+\mathrm{R}$ & 4 & \\
\hline SZC Southern June Aquilids & 88.2 & $307^{\circ} 3$ & -31.4 & 37.0 & 1.4 & 0.93 & 41.9 & 4.2 & $\mathrm{O}+\mathrm{R}$ & $3.5-7$ & \\
\hline THA November $\theta$ Aurigids & 240.5 & 92.3 & +34.7 & 33.1 & 1.1 & 0.89 & 26.4 & 5.0 & $\mathrm{O}+\mathrm{R}$ & $4-8$ & \\
\hline XRI Daytime $\xi$ Orionids & 123.7 & 98.7 & +15.9 & 42.6 & 6.8 & 0.98 & 27.3 & 1.1 & $\mathrm{R}$ & $\ldots$ & \\
\hline ZCA Daytime $\zeta$ Cancrids & 153.5 & $127^{\circ} 9$ & $+15^{\circ} .3$ & 43.0 & 4.8 & 0.99 & 18.9 & 1.4 & $\mathrm{R}$ & $\ldots$ & \\
\hline
\end{tabular}

Notes. Listed parameters are IAUMDC's three-letter code and name, solar longitude of peak activity, radiant in J2000 reference frame and geocentric speed at peak activity, orbital elements including $a, e, i$, and $T_{\mathrm{J}}$, the technique that detected the shower $(\mathrm{O}=$ optical, $\mathrm{R}=$ radar), the estimated age from Section 3.1 .2 (if available), and proposed parent body in the IAUMDC Catalog. The data spreadsheet is available at https://github.com/Yeqzids/near-sun-disruptions/blob/master/data/ streamfulldata_established.csv and a Jupyter notebook that used to prepare this table available at https://github.com/Yeqzids/near-sun-disruptions/blob/master/nb/ shower_table_established.ipynb.

a The IAUMDC catalog (version 2018 November 9) listed only radar detection of this shower, but optical detection has been reported by Jenniskens et al. (2018).

b The IAUMDC catalog gives C/2003 Q1 (SOHO) as a likely parent, but we find no published research that proposed or discussed this linkage. The orbit of C/2003 Q1 $(\mathrm{SOHO})$ is based on an extremely short arc (less than a day); therefore, the linkage is questionable.

${ }^{\mathrm{c}}$ The IAUMDC catalog gives $\mathrm{C} / 1997 \mathrm{H} 2(\mathrm{SOHO})$ and C/2009 U10 (SOHO) as likely parents, but we find no published research that proposed or discussed this linkage. Orbits of these two comets are based on very short arcs (6 days for C/1997 H2, 1 day for C/2009 U10). The linkages are therefore questionable.

2. Broadly speaking, there is a visible albeit not dramatic difference between the maps derived from the cometary ejection model and the gravitational escape ejection model, consistent with the numbers we derived in Section 2.

3. The raw inputs (the upper panel of each figure) show broken short segments: this is because some evolved streams have $>10 \%$ particles scattered into very different orbits and therefore bloat the stream width. The stream width shrinks once these highly unstable particles are ejected from the solar system.

4. The raw inputs also show appreciable scatters of age values across the $T_{\mathrm{J}}$-width space, which seems to undermine our method. However, we note that neither $T_{\mathrm{J}}$ nor stream width can be tightly constrained from observations, and that our goal is to identify broad ranges of evolutionary ages compatible with the observations. The validation, to be described below, confirms that our estimates are broadly consistent with the ones derived from stream-specific models. Therefore, the scattering is not of concern for our purpose.

5. A small but non-negligible fraction of the streams have evolved from low $T_{\mathrm{J}}$ to high $T_{\mathrm{J}}$, which we will discuss later.
Dynamics of several streams in Table 2 have been previously studied by other researchers, providing an opportunity for a sanity check. These streams are marked in both figures: Abedin et al. $(2017,2018)$ reported the age of ARI, DLT, NDA, and SDA (all originated from the 96P/Machholz complex) to be 10-20 kyr; various studies on GEM suggested an age of a few kyr (see Neslušan 2015).

We mark the locations of ARI, DLT, NDA, SDA, and GEM in Figures 4 and 5, using the stream width used by the original research (for GEM, we use the most recent visual data that has been used for some of the recent modeling work, see Arlt \& Rendtel 2006; Ryabova 2016) and $T_{\mathrm{J}}$ calculated from the mean orbit provided by IAUMDC.

At first glance, estimates using our map agree well on the age of the Geminids but underestimate the age of the 96P/ Machholz streams. The main reason for that is that Abedin et al. $(2017,2018)$ assumed continuous ejection from 96P/ Machholz, while we assume one-time ejection to mimic thermal disruption events. Assuming constant ejection rate and meteoroid delivery efficiency, the age derived from the continuous ejection model should be twice as long as the onetime ejection model, which suggests that our estimate is, in fact, in line with the numbers derived by Abedin et al. (2017, 2018). 

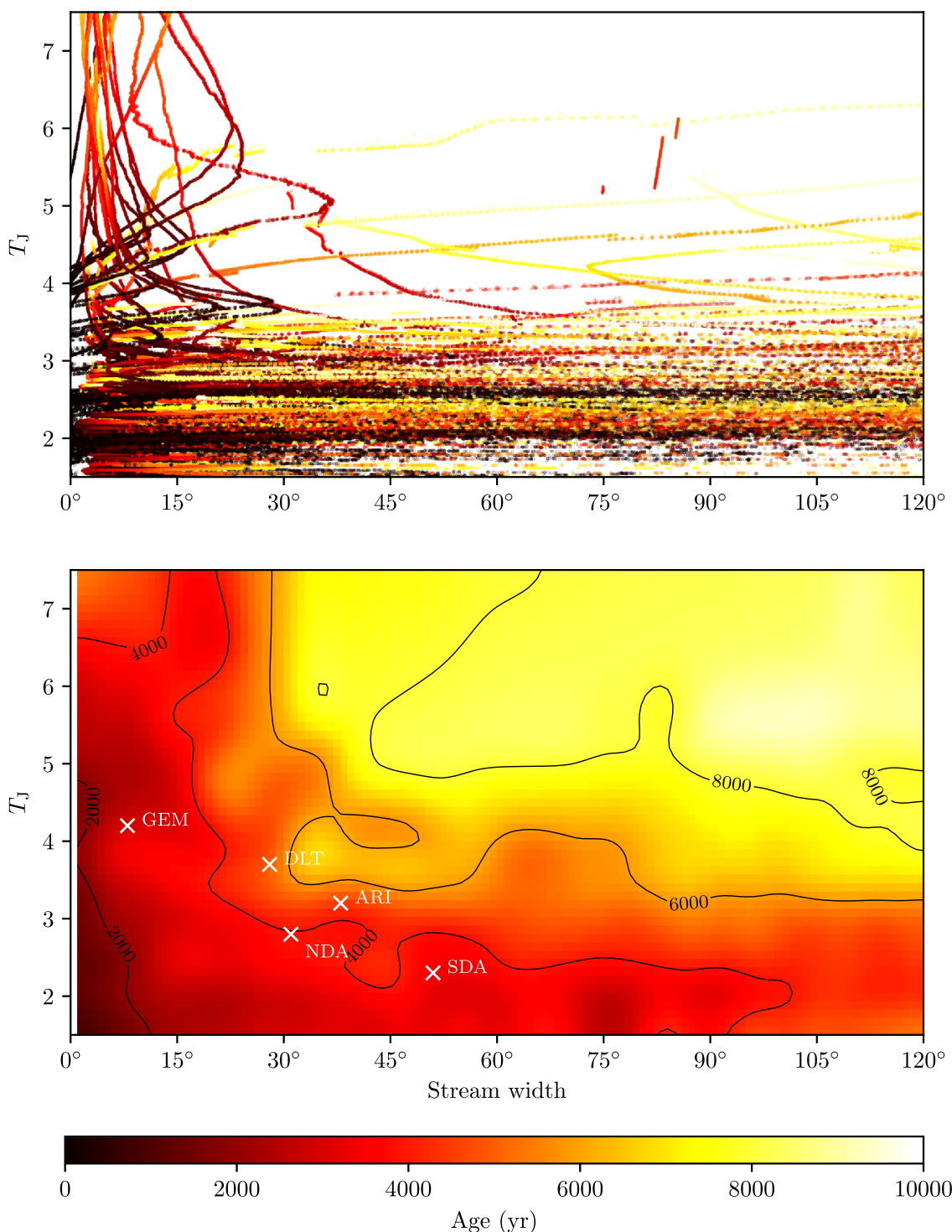

Figure 4. Raw (top) and smoothed (bottom) age-width- $T_{\mathrm{J}}$ map using cometary ejection model. The Jupyter notebook for this figure is available at https://github. com/Yeqzids/near-sun-disruptions/blob/master/nb/age_map.ipynb.

\subsubsection{Estimating the Age}

To estimate the age using the age-width- $T_{\mathrm{J}}$ map, we need the width and $T_{\mathrm{J}}$ of the stream. $T_{\mathrm{J}}$ can be readily calculated using the orbits provided by the IAUMDC catalog, but the stream width (i.e., the duration of the shower activity) is not directly provided. Therefore, we look at the original work that published these showers to obtain information on activity duration. For optical streams, the most recent measurements can be obtained from the Cameras for Allsky Meteor Surveillance (CAMS) composite shower look-up table. The most recent release of the table is version 2018-1, which can be accessed at http://cams.seti.org/ FDL/data/CAMS-ShowerLookUpTable-v2018-01.txt (Jenniskens et al. 2018). For radar streams, we use the catalog compiled by Brown et al. (2010), which is based on the meteor orbits measured by the Canadian Meteor Orbit Radar, the largest data set of its kind.

We then overlay the $\left\{\right.$ width, $\left.T_{\mathrm{J}}\right\}$ points of the streamof-interest on the age-width- $T_{\mathrm{J}}$ map, shown as Figure 6. From now on we will stick to the map generated by the gravitational escape ejection model, because it is not essentially different from the one generated by the cometary ejection model, and is more plausible for thermally driven activity. The map suggests that all these streams have an age of a couple thousand years, but as a sanity check by using the $96 \mathrm{P} / \mathrm{Machholz}$ streams discussed above has shown, these age estimates are somewhat dependent on how the particles are ejected. This will be discussed in greater detail in Section 3.2.

One curious finding is the disagreement on the width of the same stream between optical and radar data: the difference can get up to a factor of 10 in extreme cases (e.g., THA). We note that the GEM optical measurement obtained from the CAMS shower look-up table is also about seven times wider than earlier measurements shown in Ryabova (2016). There are two explanations for the difference: uncertainty in the determination of the start and end times of meteor showers may be the culprit (e.g., Brown et al. 2008, Section 4), or, the fact that optical and radar systems are sensitive to different size regimes of meteoroids. It is difficult to determine which one is more important or if they are equally important. For the latter explanation, there are known examples where the meteoroid size distribution varies across a stream (Campbell-Brown et al. 2006; 

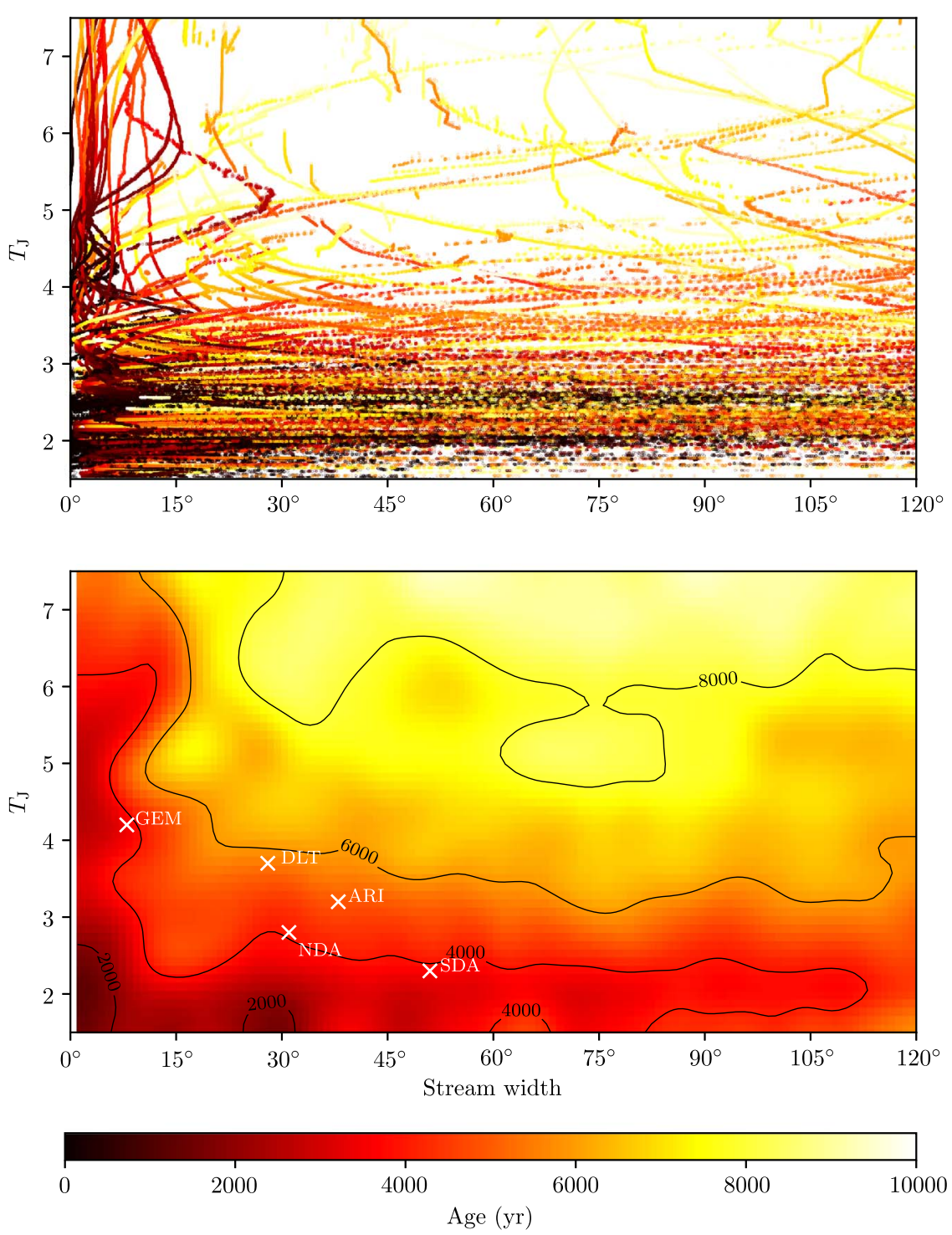

Figure 5. Raw (top) and smoothed (bottom) age-width- $T_{\mathrm{J}}$ map using gravitational escape ejection model. The Jupyter notebook for this figure is available at https:// github.com/Yeqzids/near-sun-disruptions/blob/master/nb/age_map.ipynb.

Ye et al. 2014). Fortunately, the difference does not have a dramatic impact on the age estimates. Even for streams with optical-radar width of a factor of 10, the difference between the resulting age estimates is only up to a factor of 2 .

To conclude, even after considering different modes of ejection (instantaneous versus continuous ejection) and the uncertainty in stream width, the ages of the streams in Table 2 are on the order of a few thousand years with uncertainties likely within a factor of $\sim 2$.

\subsection{Possible Biases and Implications}

If we compare the derived ages with the dispersion timescale derived in Section 2.3.3, we immediately note that the estimated ages are consistently on the longer side of the predicted dispersion timescale. So where are the young streams?

One plausible explanation is that our consideration of "established" showers introduce a bias against young, shortduration, and therefore hard-to-confirm streams. According to Figures 4 and 5, streams younger than $\sim 1 \mathrm{kyr}$ are active for less than about a day, and are thus difficult to detect and confirm unless they are strong.

Could the IAUMDC Working List contain some of the young showers? We examine the Working List and plot any qualified showers to the age-width- $T_{\mathrm{J}}$ map (Figure 7 ). There are a few candidates, such as APG $\left(3^{\circ}\right)$, DRG $\left(3^{\circ}\right)$, TOP $\left(4^{\circ}\right)$, and $\mathrm{KCT}\left(5^{\circ}\right)$, alongside many low $T_{\mathrm{J}}$ streams that are only seen by optical systems. It is difficult to say how many shortduration streams we have missed, but the possibility of bias is real. The deployment of optical and radar networks across the world (e.g., Janches et al. 2015; Pokorný et al. 2017; Jenniskens et al. 2018; Li et al. 2018) will increase the temporal coverage and orbit statistics and enhance our understanding of young streams.

Another possibility is that these streams are trapped in resonances and are therefore somewhat immune to dispersion. To test this hypothesis, we plot the semimajor axes of the streams over Figure 3, with the results shown in Figure 8. We do not find clear concentrations near the $7: 2 \mathrm{~J}$ and $3: 1 \mathrm{~J}$ resonances; in fact, as indicated by the distribution of $T_{\mathrm{J}}$, about half of these streams are decoupled from Jupiter so that 


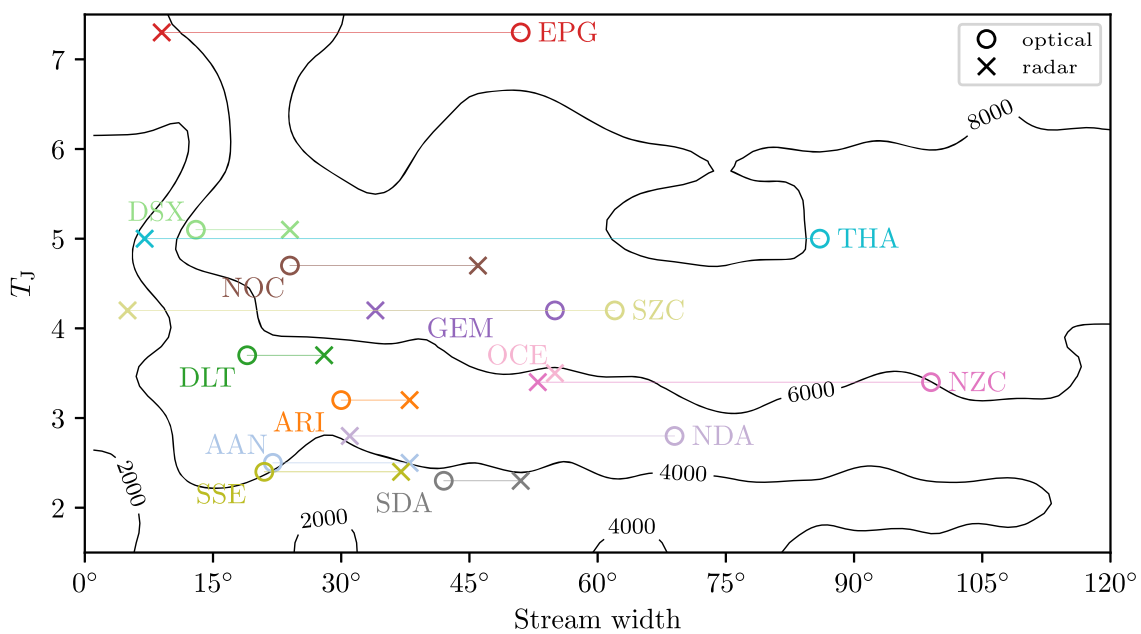

Figure 6. Sun-approaching streams with $T_{\mathrm{J}}>2$ on age-width- $T_{\mathrm{J}}$ map, assuming ejection at gravitational escape speed. To assist viewing, optical/radar data of the same stream is connected with thin lines. Note that OCE has no optical detection. The Jupyter notebook for this figure is available at https://github.com/Yeqzids/ near-sun-disruptions/blob/master/nb/age_map.ipynb.

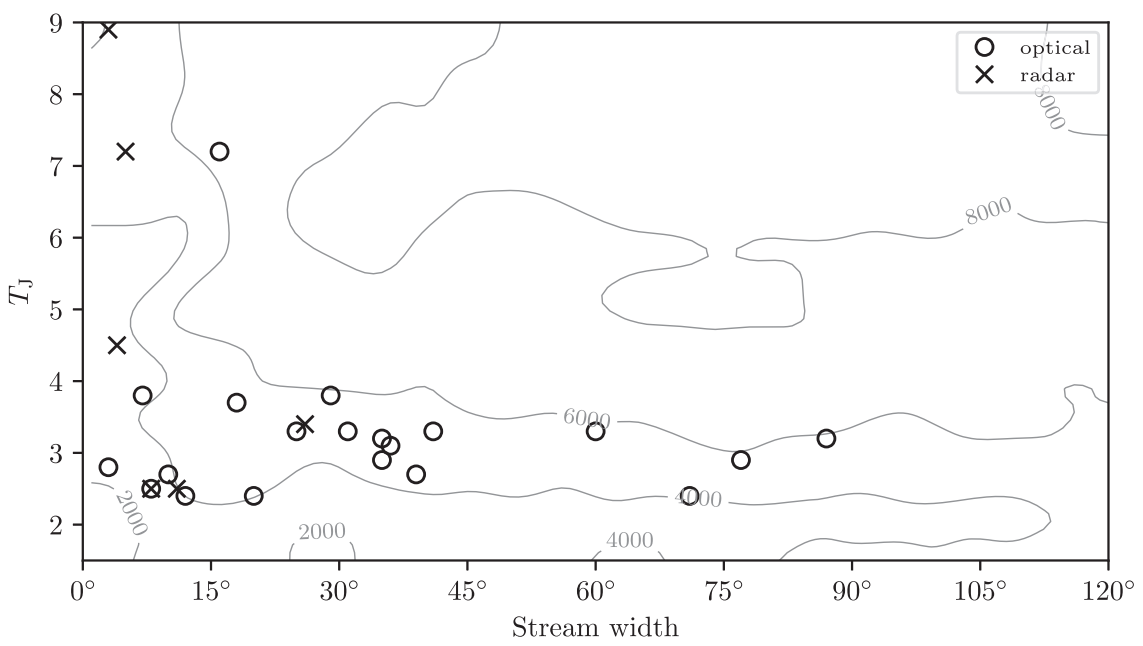

Figure 7. Same as Figure 6, but showing Working List showers. All of these showers have only either radar or optical detections. The Jupyter notebook for this figure is available at https://github.com/Yeqzids/near-sun-disruptions/blob/master/nb/age_map.ipynb.

they are immune to the perturbation from Jupiter. On a separate note, this is also consistent with the finding in Section 2.2 that objects from the inner parts of the asteroid belt are more likely to thermally disrupt than those from the outer asteroid belt.

\section{Discussion}

If we compare the model prediction made in Section 2 to the observations presented in Section 3, a clear disagreement is revealed: the model predicts that the likelihood of detecting any thermally driven stream at the Earth is (visible timescale at the Earth) $\times$ (likelihood of being visible at the Earth) $\times($ rate of disruption). By plugging in the numbers derived in Sections 2.2 and 2.3.3, this will be $40 \mathrm{yr} \times 75 \% \times 0.6 \mathrm{kyr}^{-1}=0.02$, or 1 in 50. However, observations have revealed a dozen Sunapproaching streams that are dynamically compatible with asteroids and have no known parents. If all these streams were produced by thermally driven disruptions, it would require a disruption rate of (number of streams) $\div$ (visible timescale at the Earth) $\div$ (likelihood of being visible at the Earth), or $10 \div 40 \div 75 \% \mathrm{yr}=300 \mathrm{kyr}^{-1}$, which is $\sim 500$ times higher than model prediction. Even if we exclusively focus on the streams with $T_{\mathrm{J}}>3$, which have a visible probability of $90 \%$ and a median visible timescale of $600 \mathrm{yr},{ }^{10}$ the likelihood of detection is $600 \mathrm{yr} \times 90 \% \times 0.6 \mathrm{kyr}^{-1}=0.3$. Such a disagreement clearly implies a problem with the assumptions, and the problem may be:

1. an underestimation of the rate of thermally driven disruptions from the NEO population model;

2. contamination from sources other than thermally driven disruption; or

3. a different ejection regime than assumed in our model.

Some educated guesses of the properties of the (ex-)parents may provide clues. Equation (1) shows that the stream mass is proportional to the square of the duration of activity, and it is the deterministic variable for the total stream mass, as (for short-period streams) contributions from other variables are not significant. In the highly idealized example that we discussed in Section 2.1, a complete disruption of a $500 \mathrm{~m}, 10^{11} \mathrm{~kg}$ asteroid

\footnotetext{
${ }^{10}$ Details of the calculation can be found in the Jupyter notebook, available at https://github.com/Yeqzids/near-sun-disruptions/blob/master/nb/tj_gt_ 3_streams.ipynb.
} 

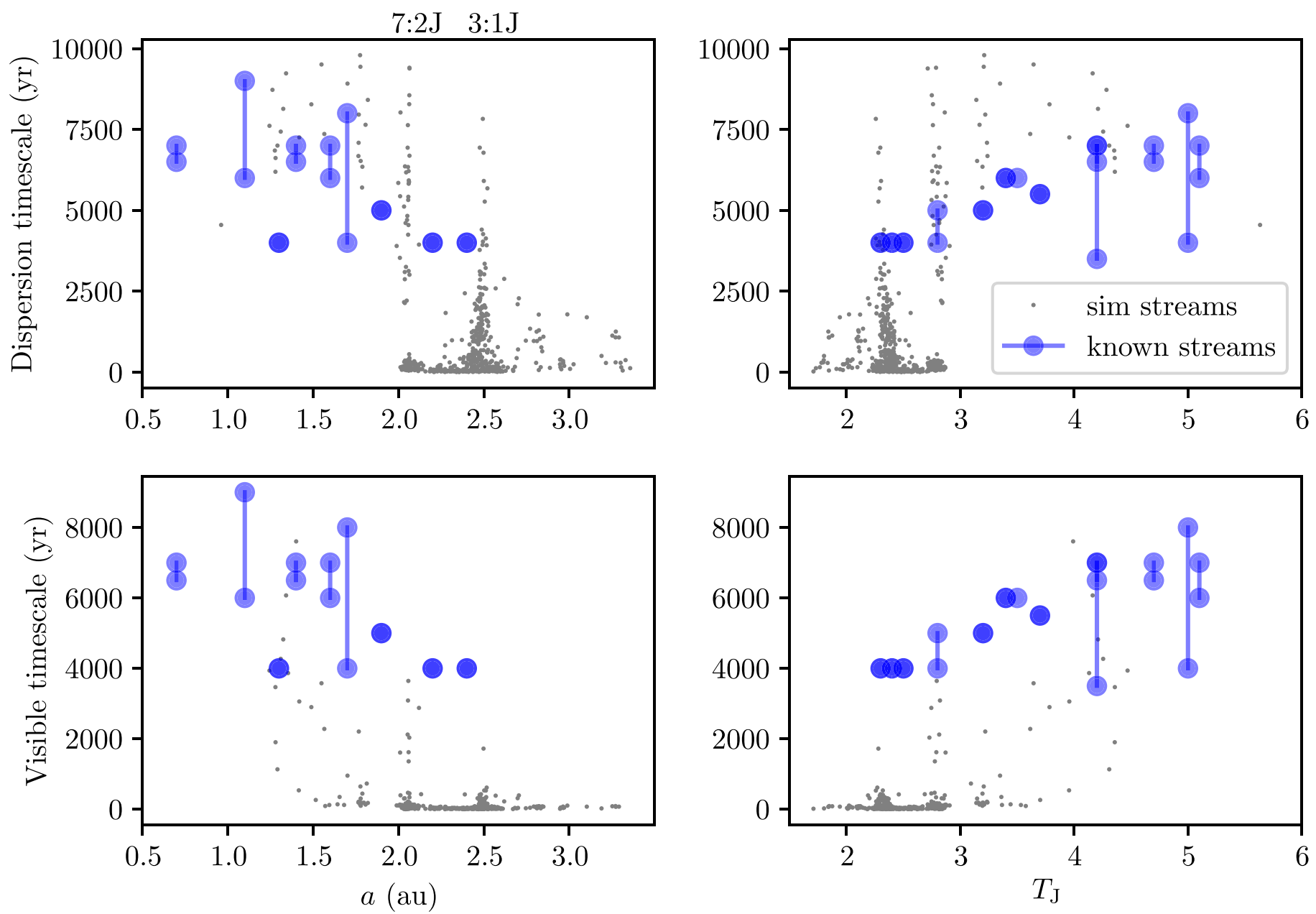

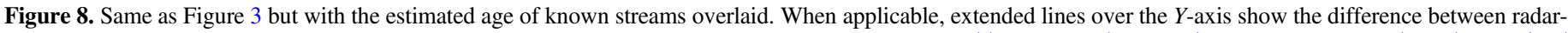

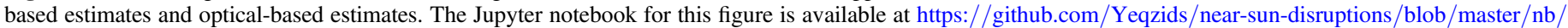
timescales_established.ipynb.

will produce a shower that lasts 5 days. A shower that lasts 10 times longer (more in line with the results in Figure 6) would require a 100 times more massive parent (i.e., $10^{13} \mathrm{~kg}$ in mass), or $2 \mathrm{~km}$ in size. For streams that are produced as a result of cometary activity instead of complete disruptions, the parents are larger in size: a close comparison might be the streams that were produced by the $10 \mathrm{~km}$ diameter comet $1 \mathrm{P} /$ Halley ( $\eta$ Aquarids and Orionids), which is collectively $3 \times 10^{13} \mathrm{~kg}$ in mass (Hughes $\&$ McBride 1989), although we acknowledge that smaller comets could also be responsible for massive streams if they are sufficiently active.

\subsection{Underestimation from the NEO Population Model}

Underestimation from the Granvik et al. (2016) model would imply that the number of thermally driven disruptions of kilometer-sized bodies over the past $\sim 10 \mathrm{kyr}$ is $1-2$ orders of magnitude more frequent than what the Granvik et al. (2016) model predicts. However, Granvik et al.'s (2016) model does otherwise agree well with the observed NEO population, and other models based on a similar modeling approach also show good agreement with the observational data (e.g., Bottke et al. 2002; Greenstreet et al. 2012). Therefore, this scenario seems unlikely.

\subsection{Contamination of Streams Produced by Other Mechanisms}

Most known meteoroid streams are produced by comets and therefore one could speculate that Sun-approaching comets may have supplied some of the streams in Table 2. Compared to thermally driven disruptions of asteroids, that will convert the entire mass into a meteoroid stream, comets are more "sustainable" and only deposit a fraction of their mass to the meteoroid stream as they orbit the Sun (unless they disrupt), and therefore need to be larger in size. However, the streams produced by comets will also be longer-lived because they will be continuously replenished. Especially comets in the LidovKozai resonance like 96P/Machholz will periodically return to low- $q$ zone (Levison \& Dones 2014), and they can therefore potentially maintain a stream for a very long time.

However, half of the streams in Table 2 are dynamically asteroidal. This scenario also results in a large number of comets that is not supported by observations: using the numbers derived in Section 2.3.3, we crudely estimate that a stream has $40 / 170-40 / 280$ or 1 in 7 to 1 in 4 chance of being detectable at any time, meaning that (statistically speaking) four to seven comets are needed to produce one detectable stream.

Another potential source of contamination comes from the streams produced at ordinary distances from the Sun that migrate into low- $q$ orbits due to the Lidov-Kozai mechanism 
and Poynting-Robertson drag, as noted by Wiegert (2008). They found that such a process can occur over a timescale of a couple thousand years, which is in the range of the ages we derived for the Sun-approaching streams. However, the same study also noted that particles of different sizes have different responses to this process: smaller particles are more sensitive to Poynting-Robert drag, while larger particles are largely immune, which implies that these "immigrants" should largely be only detectable in radio wavelengths, a prediction that is incompatible with observations.

\subsection{Prolonged Disruption Phase}

For the case of the Machholz complex, which we discussed in Section 3.1.1, we see that estimates by the instantaneous ejection model can underestimate the age of continuously replenished streams by a factor of several. The latter streams are also longer-lived because their cores will be replenished and will stay compact over time. Asteroids that have completely disintegrated will no longer be able to replenish their streams; however, if their disruption phase spans over multiple orbits, they will be able to maintain their streams longer than what our instantaneous model predicts.

In order to maintain 10 Sun-approaching streams, the visible timescale of each stream would need to be 10/0.6/ $75 \% \mathrm{kyr}^{-1}=22 \mathrm{kyr}$, which is 500 times longer than the prediction made for instantaneous ejection. Assuming the relation between visible timescale and dispersion timescale is fixed, each parent would need to "shepherd" its stream over a $10-20 \mathrm{kyr}$ timescale. The timescale shifts to the shorter end if there are fewer streams to maintain: for one stream the timescale becomes as short as $1-2 \mathrm{kyr}$.

Besides meteor observations, another observational implication of the "prolonged-disruption" theory is this: instead of a gigantic, one-time near-Sun explosion event that happens once every $\sim 2 \mathrm{kyr}$, observers are more likely to see a handful of Sun-approaching asteroids that will only release a fraction of its mass, and therefore only mildly brighten during their perihelion passages. Existing observational evidence is consistent with this theory: in the past several decades, a couple of periodic Sun-approaching objects have been discovered by the $\mathrm{SOHO}$. These objects are all designated as comets because most objects detected at such distances are Sun-grazing comets (Knight et al. 2010), even though most, if not all these periodic objects never display any coma or tail. Here we note that objects are only detectable by $\mathrm{SOHO}$ when they get to $V=8$ or brighter, because the limiting magnitude of $\mathrm{SOHO}$ is about $\mathrm{V}=8$. Therefore, these $\mathrm{SOHO}$ "comets" must either be very large or actively producing dust. However, these $\mathrm{SOHO}$ "comets" have never been detected by ground-based NEO surveys that are sensitive down to $V=21$ (Jedicke et al. 2015), which suggests that they are no larger than typical NEOs detected by surveys ( $\sim 0.1$ to a few kilometers in sizes). This also suggests that these objects are apparently inactive when away from perihelion even though they are inside the sublimation distance of water ice, the dominant volatile species in comets, seemingly implying that these are asteroids. In fact, dedicated observations of one such object, $322 \mathrm{P} / \mathrm{SOHO}$, have shown characteristics consistent with asteroids (Knight et al. 2016).

Figure 9 shows the orbital distribution of all known objects with $q<0.2$ au and $T_{\mathrm{J}}>2$, with objects found by $\mathrm{SOHO}$ and ground-based NEO surveys in different colors. Here we clearly see a transition at $0.06 \mathrm{au}$, the distance that thermally driven

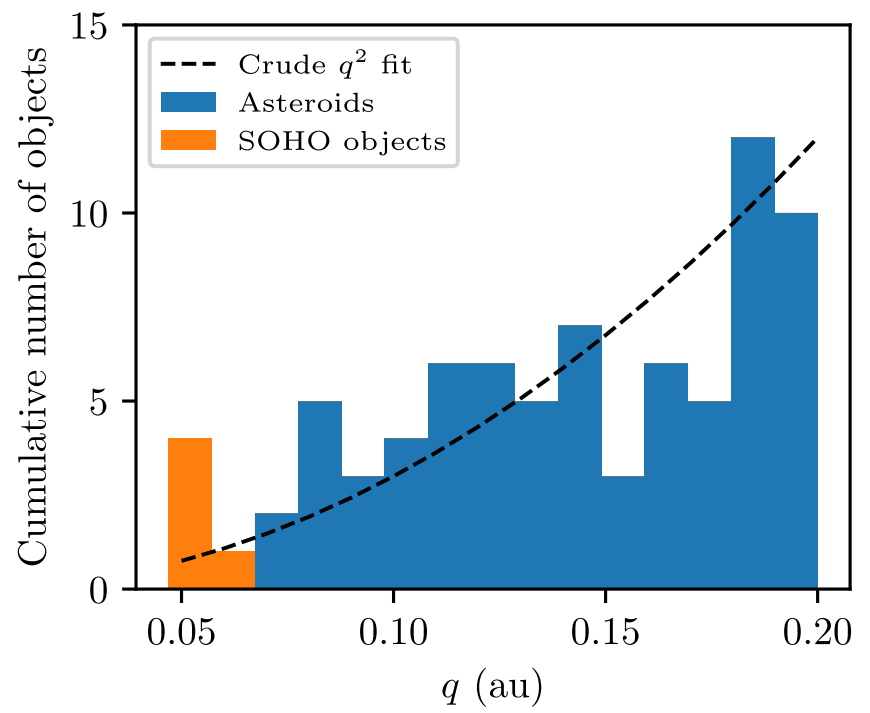

Figure 9. Distribution of perihelion distance $q$ of objects with $q<0.2$ au and $T_{\mathrm{J}}>2$. Objects found by $\mathrm{SOHO}$ (meaning that they will get to $V<8$ during perihelion) and ground-based NEO surveys are shown in different colors. Also shown is a $q^{2}$ curve that gauges the number density of objects as a function of $q$. The Jupyter notebook for this figure is available at https://github.com/ Yeqzids/near-sun-disruptions/blob/master/nb/soho_objects.ipynb.

disruption is predicted to occur by Granvik et al. (2016). We also overlay a $q^{2}$ curve to crudely match the histogram, which shows the number density stays constant throughout the region and argues against contamination from other small body populations (e.g., comets). Therefore, we conclude that at least some of the periodic SOHO "comets" might, in fact, be asteroids that are being destroyed.

The $q^{2}$ fit also provides some clues regarding the timescale of the disruption. If the timescale is short, then we should see no more than a few objects in this regime, because they are removed quite efficiently once they reach the disruption distance; if the timescale is long, then we should see a "pileup" of objects in this regime. The fact that the distribution crudely agrees with the fit suggests that the timescale is unlikely to be too much different from the derived disruption rate of $0.6 \mathrm{kyr}^{-1}$, which is also in line with the timescales we derived at the beginning of this section, although we caution that the small statistics effectively limits our discussion to the order-of-magnitude level.

\section{Conclusions}

It is quite possible that Sun-approaching meteoroid streams are fed by multiple sources. Some of $2<T_{\mathrm{J}}<3$ streams could have been produced by short-period comets instead of asteroids, while the possibility that thermally driven disruption of asteroids could be a relatively lengthy process may also contribute to the number of Sun-approaching streams being seen.

The hypothesis that thermally driven disruption is a lengthy process also implies that such a process might be observable on the current Sun-approaching asteroids, with $\mathrm{SOHO}$ objects as prominent examples. Granvik et al. (2016) predicts that thermally driven disruption can occur on small, dark asteroids at slightly larger distances, possibly up to $\sim 0.4 \mathrm{au}$. The brightening effect on asteroids at a few tenths of an astronomical unit will be less pronounced and would be difficult for $\mathrm{SOHO}$ and other coronagraphs to detect. 
Ground-based observers, on the other hand, could have sufficient sensitivity to detect any brightening but have difficulties with small solar elongations. However, space probes operating close to or inside of the orbit of Mercury such as MESSENGER, BepiColombo, or Parker Solar Probe will have a chance to test this hypothesis.

Our work offers some lessons for understanding solid-body disruptions in other planetary systems. Although we have yet to be able to directly image small bodies in exoplanetary systems, signatures of disrupting small bodies have been found in a variety of planetary systems (e.g., Montgomery \& Welsh 2012; Kiefer et al. 2014; Vanderburg et al. 2015; Rappaport et al. 2016; Xu et al. 2016, 2018). The direct exoplanetary equivalents of the debris streams investigated in this work (likely at the level of $10^{-10}-10^{-12}$ Earth mass) is beyond the detection capability of current techniques, but disruptions of larger bodies that are potentially detectable by current techniques should be governed by the same physics. Our finding of prolonged disintegration of Sun/star-approaching rocky bodies suggests that the observing window for such events is long, and may partly explain the common occurrence of such phenomenon in exoplanetary systems.

The authors thank Peter Brown for his contribution to an early version of the draft and Paul Wiegert for access to computational resource. Q.-Z.Y. is supported by the GROWTH project funded by the National Science Foundation under grant No. 1545949. M.G. is supported by grant No. 299543 from the Academy of Finland. This work was made possible by the facilities of the Shared Hierarchical Academic Research Computing Network (SHARCNET: www.sharcnet.ca) and Compute/Calcul Canada. We extend our thanks to the American Astronomical Society's Division for Planetary Science for holding its annual meeting, which provides an opportunity for the authors to meet in person and to fight their procrastination.

Software: Astropy (Robitaille et al. 2013), Jupyter Notebooks (Kluyver et al. 2016), Matplotlib (Hunter 2007), MERCURY6 (Chambers 1999), NumPy (Walt et al. 2011).

\section{ORCID iDs}

Quanzhi Ye (叶泉志) (1) https://orcid.org/0000-0002-4838-7676 Mikael Granvik (ib https://orcid.org/0000-0002-5624-1888

\section{References}

Abedin, A., Wiegert, P., Janches, D., et al. 2018, Icar, 300, 360 Abedin, A., Wiegert, P., Pokorný, P., \& Brown, P. 2017, Icar, 281, 417 Arlt, R., \& Rendtel, J. 2006, MNRAS, 367, 1721

Blaauw, R. C., Campbell-Brown, M. D., \& Weryk, R. J. 2011, MNRAS, 414, 3322

Bottke, W. F., Morbidelli, A., Jedicke, R., et al. 2002, Icar, 156, 399

Brown, P., \& Jones, J. 1998, Icar, 133, 36

Brown, P., Weryk, R. J., Wong, D. K., \& Jones, J. 2008, EM\&P, 102, 209

Brown, P., Wong, D. K., Weryk, R. J., \& Wiegert, P. 2010, Icar, 207, 66

Bruzzone, J., Brown, P., Weryk, R., \& Campbell-Brown, M. 2014, MNRAS, 446, 1625

Campbell-Brown, M., Vaubaillon, J., Brown, P., Weryk, R. J., \& Arlt, R. 2006, A\&A, 451, 339

Carry, B. 2012, P\&SS, 73, 98

Chambers, J. E. 1999, MNRAS, 304, 793

Cremonese, G., Fulle, M., Marzari, F., \& Vanzani, V. 1997, A\&A, 324, 770

Crifo, J. F., \& Rodionov, A. V. 1997, Icar, 127, 319

Denning, W. F. 1893, Natur, 47, 226

Drummond, J. D. 1981, Icar, 45, 545
Fu, H., Jedicke, R., Durda, D. D., Fevig, R., \& Scotti, J. V. 2005, Icar, 178, 434 Fulle, M. 2004, in Comets II, ed. M. C. Festou, H. U. Keller, \& H. A. Weaver (Tucson, AZ: Univ. Arizona Press), 565

Granvik, M., Morbidelli, A., Jedicke, R., et al. 2016, Natur, 530, 303

Granvik, M., Morbidelli, A., Jedicke, R., et al. 2018, Icar, 312, 181

Greenstreet, S., Ngo, H., \& Gladman, B. 2012, Icar, 217, 355

Hsieh, H. H., \& Haghighipour, N. 2016, Icar, 277, 19

Hughes, D. W., \& McBride, N. 1989, MNRAS, 240, 73

Hui, M.-T., \& Li, J. 2017, AJ, 153, 23

Hui, M.-T., Ye, Q.-Z., Knight, M., Battams, K., \& Clark, D. 2015, ApJ, 813, 73 Hunter, J. D. 2007, CSE, 9, 90

Janches, D., Brown, P., Jenniskens, P., et al. 2018, Trans. IAU, XXXA, 1

Janches, D., Close, S., Hormaechea, J. L., et al. 2015, ApJ, 809, 36

Jedicke, R., Granvik, M., Micheli, M., et al. 2015, in Asteroids IV, ed.

P. Michel, F. E. DeMeo, \& W. F. Bottke (Tucson, AZ: Univ. Arizona Press), 795

Jenniskens, P. 2017, P\&SS, 143, 116

Jenniskens, P., Baggaley, J., Crumpton, I., et al. 2018, P\&SS, 154, 21

Jenniskens, P., Nénon, Q., Albers, J., et al. 2016a, Icar, 266, 331

Jenniskens, P., Nénon, Q., Gural, P. S., et al. 2016b, Icar, 266, 384

Jewitt, D., Hsieh, H., \& Agarwal, J. 2015, in Asteroids IV, ed. P. Michel,

F. E. DeMeo, \& W. F. Bottke (Tucson, AZ: Univ. Arizona Press), 221

Jones, J., Poole, L. M. G., \& Webster, A. R. 2016, MNRAS, 455, 3424

Jopek, T. J. 1993, Icar, 106, 603

Jopek, T. J., \& Jenniskens, P. M. 2011, in Meteoroids: The Smallest Solar System Bodies, ed. W. J. Cooke et al. (Huntsville, AL: NASA), 7

Jopek, T. J., \& Kan̆uchová, Z. 2014, Meteoroids, 2013, 353

Jopek, T. J., \& Kaňuchová, Z. 2017, P\&SS, 143, 3

Kiefer, F., Lecavelier des Etangs, A., Boissier, J., et al. 2014, Natur, 514, 462

Kluyver, T., Ragan-Kelley, B., Pérez, F., et al. 2016, in Positioning and Power in Academic Publishing: Players, Agents and Agendas, ed. F. Loizides \& B. Schmidt (Amsterdam: IOS Press), 87

Knight, M. M., A’Hearn, M. F., Biesecker, D. A., et al. 2010, AJ, 139, 926

Knight, M. M., Fitzsimmons, A., Kelley, M. S. P., \& Snodgrass, C. 2016, ApJL, 823, L6

Koschack, R., \& Rendtel, J. 1990, JIMO, 18, 119

Levison, H. F., \& Dones, L. 2014, in Encyclopedia of the Solar System (3rd ed.; Amsterdam: Elsevier), 705

Levison, H. F., \& Duncan, M. J. 1994, Icar, 108, 18

Li, G., Ning, B., Li, A., et al. 2018, Earth Planet. Phys., 2, 15

McIntosh, B. A., \& Jones, J. 1988, MNRAS, 235, 673

Montgomery, S. L., \& Welsh, B. Y. 2012, PASP, 124, 1042

Moorhead, A. V. 2016, MNRAS, 455, 4329

Neslušan, L. 2015, CoSka, 45, 60

Neslušan, L., \& Hajduková, M. 2014, A\&A, 566, A33

Olsson-Steel, D. 1987, MNRAS, 226, 1

Plavec, M. 1950, Natur, 165, 362

Pokorný, P., Janches, D., Brown, P. G., \& Hormaechea, J. L. 2017, Icar, 290 , 162

Rappaport, S., Gary, B. L., Kaye, T., et al. 2016, MNRAS, 458, 3904

Robitaille, T. P., Tollerud, E. J., Greenfield, P., et al. 2013, A\&A, 558, A33

Rotundi, A., Sierks, H., Della Corte, V., et al. 2015, Sci, 347, aaa3905

Ryabova, G. O. 1999, SoSyR, 33, 224

Ryabova, G. O. 2016, MNRAS, 456, 78

Ryabova, G. O. 2017, P\&SS, 143, 125

Ryabova, G. O., \& Rendtel, J. 2018, MNRAS, 475, L77

Southworth, R. B., \& Hawkins, G. S. 1963, SCoA, 7, 261

Stoer, J., \& Bulirsch, R. 2013, Introduction to Numerical Analysis, Vol. 12 (New York: Springer)

Vanderburg, A., Johnson, J. A., Rappaport, S., et al. 2015, Natur, 526, 546

Vereš, P., Kornoš, L., \& Tóth, J. 2011, MNRAS, 412, 511

Walt, S. v. d., Colbert, S. C., \& Varoquaux, G. 2011, CSE, 13, 22

Whipple, F. L. 1951, ApJ, 113, 464

Whipple, F. L. 1983, IAUC, 3881

Wiegert, P. A. 2008, EM\&P, 102, 15

Williams, I. P., Ryabova, G. O., Baturin, A. P., \& Chernitsov, A. M. 2004, MNRAS, 355, 1171

Williams, I. P., \& Wu, Z. 1993, MNRAS, 262, 231

Xu, S., Jura, M., Dufour, P., \& Zuckerman, B. 2016, ApJL, 816, L22

Xu, S., Su, K. Y. L., Rogers, L. K., et al. 2018, ApJ, 866, 108

Ye, Q., Wiegert, P. A., Brown, P. G., Campbell-Brown, M. D., \& Weryk, R. J. 2014, MNRAS, 437, 3812

Ye, Q.-Z. 2018, P\&SS, 164, 7

Ye, Q.-Z., Brown, P. G., \& Pokorný, P. 2016a, MNRAS, 462, 3511

Ye, Q.-Z., Brown, P. G., \& Wiegert, P. A. 2016b, ApJL, 818, L29

Ye, Q.-Z., Hui, M.-T., Brown, P. G., et al. 2016c, Icar, 264, 48 